\title{
Subthreshold dynamics of a single neuron from a Hamiltonian perspective
}

\author{
M. T. Wilson* and D. A. Steyn-Ross \\ Department of Engineering, University of Waikato, Private Bag 3105, Hamilton 3240, New Zealand \\ (Received 8 July 2008; revised manuscript received 18 September 2008; published 4 December 2008)
}

\begin{abstract}
We use Hamilton's equations of classical mechanics to investigate the behavior of a cortical neuron on the approach to an action potential. We use a two-component dynamic model of a single neuron, due to Wilson, with added noise inputs. We derive a Lagrangian for the system, from which we construct Hamilton's equations. The conjugate momenta are found to be linear combinations of the noise input to the system. We use this approach to consider theoretically and computationally the most likely manner in which such a modeled neuron approaches a firing event. We find that the firing of a neuron is a result of a drop in inhibition, due to a temporary increase in negative bias of the mean noise input to the inhibitory control equation. Moreover, we demonstrate through theory and simulation that, on average, the bias in the noise increases in an exponential manner on the approach to an action potential. In the Hamiltonian description, an action potential can therefore be considered a result of the exponential growth of the conjugate momenta variables pulling the system away from its equilibrium state, into a nonlinear regime.
\end{abstract}

DOI: 10.1103/PhysRevE.78.061908

PACS number(s): 87.19.11, 05.10.Gg, 87.10.Mn

\section{INTRODUCTION}

Path-integral formulations of dynamical systems have the potential to shed light on the physical behavior of complex systems that may not be obtainable through direct simulation of a set of dynamical equations. Often, a Lagrangian approach is taken, in which one applies the principle of least action, namely, that the most likely path taken by a system is the one that minimizes the action. A Lagrangian approach is naturally suited to problems that focus on the most likely trajectories of dynamical systems which contain a random component, such as the dynamics of neurons.

Recently, Paninski has formulated a single neuron system, specifically an integrate-and-fire model, in terms of a Lagrangian, and demonstrated that this formalism can successfully predict the most likely behavior of the neuron's membrane potential between firing events [1]. This has been tested against experimental data. Badel et al. have carried out a similar theoretical analysis with a linear two-component integrate-and-fire neuron to predict most-likely membrane potential trajectories on the approach to the firing threshold [2]; they have developed this approach to analyze the behavior of voltage-gated subthreshold ion currents in the vicinity of a spike, and compared with numerical simulations of a nonlinear neuron [3]. Ingber [4] has formulated interactions between neurons in terms of path integral approaches, with application to short-term memory.

Such techniques should have wide applicability. For example, Pospischil et al. [5] have studied the changes in excitatory and inhibitory synaptic conductance on the approach to an action potential event experimentally and numerically, and Rudolph et al. [6] have studied changes in membrane potential and conductance. They have shown that a drop in the inhibitory conductance, due to random fluctuations, is what drives the formation of an action potential. Rudolph et al. have also shown similar changes in membrane conduc-

\footnotetext{
*m.wilson@waikato.ac.nz
}

tances on the transition between "down" and "up" states of slow-wave sleep [6]. The time course of such conductance changes, being a "most-likely" situation, should be suited for study with a path-integral technique.

Additionally, the behavior of fluctuations in membrane potential on the approach to an action potential have been analyzed by Steyn-Ross et al. [7] using a two-component neuron model due to Wilson [8]. In particular, Steyn-Ross et al. showed that an action potential can be considered as a growth of a small fluctuation into a macroscopic event. This "most likely" trajectory of a neuron's membrane potential on the approach to an action potential should also be tractable through a path integral approach.

In this paper, we focus on the Hamiltonian formalism of a dynamical system. Hamilton's equations are related to the Lagrangian (path integral) approach, and often considered alongside the Lagrangian method, but the physics of a system is expressed in a different way, as a set of coupled firstorder differential equations in phase space. Since the two approaches are both formally exact, the Hamiltonian method cannot, in principle, provide any new solution that is not obtainable through the Lagragian method, and we emphasize here that we do not consider it to be generally advantageous over other approaches. However, Hamilton's equations can often provide a physical framework by which to understand the behavior of systems [9]. The Hamiltonian approach constructs "canonical momenta" conjugate to each state variable, and in practice these momenta often represent physical quantities. We find this is true for a single neuron system. For example, Ingber has used canonical momenta for a manyneuron system as a way of describing an electroencephalogram [10].

In this paper, we use Hamilton's equations to provide a physical explanation of the results of Pospischil et al. [5] and Rudolph et al. [6], specifically that the bias in the random driving of the neuron system on the approach to an action potential changes in an exponential manner. However, the Hamiltonian description of this system has limitations, and we do not suggest that it will be more useful than path integral methods for predicting most-likely time courses of neu- 
rons (e.g., that of Paninski which predicts well the subthreshold membrane potential between spike events [1]).

We first present a generalized Lagrangian description of a stochastic system, and write this in Hamiltonian form. We then place the two-component neuron model of Wilson [8] into this form and discuss its numerical implementation. We then "solve" the Hamiltonian description by simulation, with particular emphasis on the approach to an action potential, and compare the results with direct simulations of the neuron model. We comment specifically on the physical meaning of the conjugate momenta.

\section{THEORY}

\section{A. Lagrangian}

Consider the stochastic process given by the equation

$$
\frac{d \vec{x}}{d t}=\vec{f}(\vec{x}, t)+\vec{v}(t)
$$

where $\vec{x}$ is a multidimensional state vector of the system, $\vec{f}$ is an operator, and $\vec{v}$ represents the random input to the system and $t$ is time. Here the random input vector $\vec{v}$ has the same dimensions as $d \vec{x} / d t$. We start by considering a discrete form, most suitable for modeling, but take the limit of small time steps to derive a Lagrangian for the system. In terms of discrete time steps of size $\Delta t$, we can write this as the process

$$
\Delta \vec{x}=\vec{f}(\vec{x}, t) \Delta t+\sigma_{0} \vec{\xi}(t) \sqrt{\Delta t}
$$

where $\sigma_{0}$ is a matrix and the noise terms $\vec{\xi}$ are dimensionless uncorrelated Gaussian noise with mean zero and variance unity:

$$
\left\langle\xi_{i}(t) \xi_{j}\left(t^{\prime}\right)\right\rangle=\delta_{i j} \delta_{t t^{\prime}}
$$

Here $\delta$ is the Kronecker delta and the average is taken over all time steps. In this interpretation of the stochastic process we use the dimensioned matrix $\sigma_{0}$ to scale the dimensionless noise terms $\vec{\xi}(t)$; its $i$ th row carries dimensions of $\left[x_{i}\right]$ per square-root time. We can map back to continuous time by taking the limit $\Delta t \rightarrow 0$ whereupon Eqs. (2.1) and (2.2) imply that the statistics of $\vec{v}$ follow:

$$
\left\langle\vec{v}(t) \vec{v}^{T}\left(t^{\prime}\right)\right\rangle=I \sigma_{0} \sigma_{0}^{T} \delta\left(t-t^{\prime}\right),
$$

where $I$ is the identity matrix and $\delta$ now signifies the Dirac delta function. The superscript $T$ denotes the transpose, and the average is taken over continuous time. In terms of dimensions, we recall that $v_{i}$ carries dimensions of $\left[x_{i}\right]$ per time, the $i$-th row of $\sigma_{0}$ dimensions $\left[x_{i}\right]$ per square-root time, and the Dirac $\delta$ function dimensions of inverse time, making Eq. (2.4) dimensionally consistent; specifically the $i j$ component carries dimensions of $\left[x_{i}\right]\left[x_{j}\right]$ per unit time squared.

We can construct a Lagrangian for this system following Paninski [1]. The probability of a given series of random numbers $\vec{\xi}(t)$ (where $t$ denotes a discrete time index) is given by

$$
p \propto \Pi_{t} \exp \left[-\frac{1}{2} \vec{\xi}^{T}(t) \vec{\xi}(t)\right]=\exp \left[-\frac{1}{2} \sum_{t} \vec{\xi}^{T}(t) \vec{\xi}(t)\right]
$$

We identify the (dimensionless) action $S$ as the negative of the exponent. Substituting for $\vec{\xi}$ from Eq. (2.2) we obtain the action $S$ as

$$
\begin{aligned}
S=\frac{1}{2} \sum_{t} \vec{\xi}^{T}(t) \vec{\xi}(t)= & \frac{1}{2 \Delta t} \sum_{t}[\Delta \vec{x}(t)-\vec{f}(t) \Delta t]^{T} \sigma_{0}^{-1 T} \sigma_{0}^{-1}[\Delta \vec{x}(t) \\
& -\vec{f}(t) \Delta t] .
\end{aligned}
$$

Writing $\Sigma^{2}$ as the symmetric matrix $\sigma_{0} \sigma_{0}^{T}$, we can take the limit $\Delta t \rightarrow 0$ to obtain

$$
S=\frac{1}{2} \int_{t} d t(\dot{\vec{x}}-\vec{f})^{T} \Sigma^{-2}(\dot{\vec{x}}-\vec{f}),
$$

where $\dot{\vec{x}}=\lim _{\Delta t \rightarrow 0}(\Delta \vec{x} / \Delta t)$. Since $S=\int_{t} d t L(\vec{x}, \dot{\vec{x}}, t)$, we recognize the Lagrangian must be given by

$$
L=\frac{1}{2}(\dot{\vec{x}}-\vec{f})^{T} \Sigma^{-2}(\dot{\vec{x}}-\vec{f})
$$

This assumes that $\Sigma^{2}$ is an invertible matrix [in essence that noise enters in all components of Eq. (2.1)].

\section{B. Euler-Lagrange equation}

We can proceed from the Lagrangian of Eq. (2.8) to construct the Euler-Lagrange equation for the system. This denotes the most likely path for the system. We follow Paninski [1], but generalize to $N$ dimensions. Using $x_{k}$ to denote the $k$ th dimension of $\vec{x}$, the $N$ Euler-Lagrange equations are

$$
\frac{d}{d t}\left(\frac{\partial L}{\partial \dot{x}_{k}}\right)=\frac{\partial L}{\partial x_{k}}
$$

Substituting the Lagrangian $L$ from Eq. (2.8) into these equations, and using $\partial \dot{x}_{i} / \partial \dot{x}_{k}=\delta_{i k}$, we obtain the $N$ equations

$$
\begin{aligned}
\Sigma_{k j}^{-2}\left(\ddot{x}_{j}-\dot{f}_{j}\right)+\left(\ddot{x}_{i}-\dot{f}_{i}\right) \Sigma_{i k}^{-2}= & -\frac{\partial f_{i}}{\partial x_{k}} \Sigma_{i j}^{-2}\left(\dot{x}_{j}-f_{j}\right) \\
& -\left(\dot{x}_{i}-f_{i}\right) \Sigma_{i j}^{-2} \frac{\partial f_{j}}{\partial x_{k}},
\end{aligned}
$$

where summation is assumed over repeated indices, and a dot represents a full differentiation with respect to time.

To proceed we note that, by the chain rule

$$
\dot{f}_{j}=\frac{\partial f_{j}}{\partial t}+\frac{\partial f_{j}}{\partial x_{k}} \dot{x}_{k}+\frac{\partial f_{j}}{\partial \dot{x}_{k}} \ddot{x}_{k}
$$

Assuming further that the Lagrangian is an explicit function of neither $\dot{\vec{x}}$ nor $t$ (as is often the case for neuron models) i.e., $\vec{f}=\vec{f}(\vec{x})$, only the second term on the right-hand side of Eq. (2.11) remains, and we have 


$$
\dot{f}_{j}=\frac{\partial f_{j}}{\partial x_{k}} \dot{x}_{k},
$$

from which Eq. (2.10) becomes

$$
\begin{aligned}
& \Sigma_{k j}^{-2}\left(\ddot{x}_{j}-\frac{\partial f_{j}}{\partial x_{l}} \dot{x}_{l}\right)+\left(\ddot{x}_{i}-\frac{\partial f_{i}}{\partial x_{l}} \dot{x}_{l}\right) \Sigma_{i k}^{-2} \\
& \quad=-\frac{\partial f_{i}}{\partial x_{k}} \Sigma_{i j}^{-2}\left(\dot{x}_{j}-f_{j}\right)-\left(\dot{x}_{i}-f_{i}\right) \Sigma_{i j}^{-2} \frac{\partial f_{j}}{\partial x_{k}} .
\end{aligned}
$$

\section{Small fluctuations}

The set of equations (2.13) will be in general difficult to solve. However, if we consider the case of small fluctuations about an equilibrium state we can linearize the function $\vec{f}$ by writing $\vec{x}$ with reference to its long-term average, to give us $\vec{f}(\vec{x})=M \vec{x}$, where $M$ is a matrix, and we have repositioned our origin to be at the equilibrium point (i.e., $\vec{x}=\overrightarrow{0}$ denotes the equilibrium point). In this form, the Euler-Lagrange equations reduce to

$$
\begin{aligned}
& \Sigma_{k j}^{-2}\left(\ddot{x}_{j}-M_{j l} \dot{x}_{l}\right)+\left(\ddot{x}_{i}-M_{i l} \dot{x}_{l}\right) \Sigma_{i k}^{-2} \\
& \quad=-M_{i k} \Sigma_{i j}^{-2}\left(\dot{x}_{j}-M_{j l} x_{l}\right)-\left(\dot{x}_{i}-M_{i l} x_{l}\right) \Sigma_{i j}^{-2} M_{j k} .
\end{aligned}
$$

In matrix form, this gives

$$
\begin{aligned}
\Sigma^{-2} \ddot{\vec{x}}-\Sigma^{-2} M \dot{\vec{x}}+\left(\Sigma^{-2}\right)^{T} \ddot{\vec{x}}-\left(\Sigma^{-2}\right)^{T} M \dot{\vec{x}} \\
\quad=-M^{T} \Sigma^{-2} \dot{\vec{x}}+M^{T} \Sigma^{-2} M \vec{x}-M^{T}\left(\Sigma^{-2}\right)^{T} \dot{\vec{x}}+M^{T}\left(\Sigma^{-2}\right)^{T} M \vec{x},
\end{aligned}
$$

which can be rearranged as

$$
Y \ddot{\vec{x}}+\left(-Y M+M^{T} Y\right) \dot{\vec{x}}-\left(M^{T} Y M\right) \vec{x}=0,
$$

where $Y=\Sigma^{-2}+\left(\Sigma^{-2}\right)^{T} \quad\left(=2 \Sigma^{-2}\right.$, since $\Sigma^{2}$ is symmetric $)$. Furthermore, if we assume solutions of the form $\vec{x}$ $=c^{\vec{k}} \exp \left(\lambda^{(k)} t\right)$ where $k$ denotes the $k$ th solution, we have $\dot{\vec{x}}$ $=\lambda^{(k)} \vec{x}$ and $\ddot{\vec{x}}=\lambda^{(k) 2} \vec{x}$, giving the secular equations

$$
\left(-M^{T}-\lambda^{(k)} I\right) Y\left(M-\lambda^{(k)} I\right) \overrightarrow{c^{k}}=\overrightarrow{0} .
$$

where $I$ is the identity matrix.

The matrix of Eq. (2.17) is related to the power spectrum of a system of the form of Eq. (2.1). As described by Wilson et al. [11], the power spectrum $S(\omega)$ of such a system, following the method of Chaturvedi et al. [12], is

$$
S(\omega) \propto(M+i \omega I)^{-1} Y^{-1}\left(M^{T}-i \omega I\right)^{-1},
$$

which we recognize as the inverse of the matrix of Eq. (2.17), where $\lambda \rightarrow-i \omega$. Note, however, the different interpretations of the matrix; in Eq. (2.17) it is part of a secular equation, and has specific (complex) solutions for $\lambda^{(k)}$, whereas in Eq. (2.18) the expression applies for any real $\omega$, and gives resonances where $-i \omega$ approaches $\lambda^{(k)}$. For example, consider the case when the real part of an eigenvalue $\lambda^{(k)}$ is zero. Any eigenvalue would give a zero determinant for the matrix of Eq. (2.17) and therefore poles in the matrix $S(\omega)$ at angular frequencies $\pm \omega$ given by the imaginary part of $\lambda^{(k)}$. When the real part of the eigenvalue is zero, these poles correspond to undamped resonances of the system at this angular frequency.

\section{Solution to the Euler-Lagrange equation}

Equation (2.17) must be solved to provide the solutions to the Euler-Lagrange equations. Note that this is an $N$-dimensional matrix equation, of the form $R\left(\lambda^{(k)}\right) \overrightarrow{c^{k}}=\overrightarrow{0}$, where

$$
R=\left(-M^{T}-\lambda^{(k)} I\right) Y\left(M-\lambda^{(k)} I\right) .
$$

By inspection, one solution of the equation can be found by solving

$$
\left(M-\lambda^{(k)} I\right) \overrightarrow{c^{k}}=\overrightarrow{0}
$$

from which we recognize that $\overrightarrow{c^{k}}$ is an eigenvector of the matrix $M$ with eigenvalue $\lambda^{(k)}$. (In general, the eigenvalues can be complex.) We expect $N$ such solutions from an $N$-dimensional matrix.

Further inspection of Eq. (2.19) reveals a second set of solution vectors $\vec{C}^{k}$ exists where $Y\left(M-\lambda^{(k)} I\right) \vec{C}^{k}$ is an eigenvector of $M^{T}$ with eigenvector $-\lambda^{(k)}$. In general, if $\overrightarrow{u^{k}}$ are eigenvectors of a matrix $A$ with eigenvalues $\mu^{k}$, then the eigenvectors of the matrix $A^{T}$ are given by $\overrightarrow{v^{k}}$ with the same eigenvalues $\mu^{k}$, where $\overrightarrow{v^{k}}$ is the $k$ th "reciprocal-lattice" vector of the set $\left\{\overrightarrow{u^{k}}\right\}$ : that is, $\overrightarrow{v^{k}}$ is perpendicular in $N$-dimensional space to all $\vec{u}$, where $j \neq k$. This means that our second set of solutions $\vec{C}^{k}$ is related to the "reciprocal-lattice" vectors of the first set by

$$
\overrightarrow{d^{k}}=Y\left(M+\lambda^{(k)} I\right) \vec{C}^{k},
$$

where the set of $N$ vectors $\left\{\overrightarrow{d^{k}}\right\}$ are the reciprocal-lattice vectors of the set $\left\{\overrightarrow{c^{k}}\right\}$, and $\lambda^{(k)}$ are their associated eigenvalues. In this equation the $\lambda^{(k)}$ carry a positive sign so they are the same $\lambda^{(k)}$ as for the set of vectors $\{\vec{c}\}$; however, it is the negative of these that satisfies the secular equation for the second set of modes. Equation (2.21) can be solved to give the solutions

$$
\vec{C}^{k}=\left(M+\lambda^{(k)} I\right)^{-1} Y^{-1} \vec{d}^{k} .
$$

The solution of the Euler-Lagrange equations (2.14) is then a linear combination of all the eigensolutions

$$
\vec{x}=\sum_{k=1}^{N} a_{k} \vec{c}^{\vec{k}} \exp \lambda^{(k)} t+\sum_{k=1}^{N} b_{k}\left(M+\lambda^{(k)} I\right)^{-1} Y^{-1} \vec{d}^{k} \exp \left(-\lambda^{(k)} t\right),
$$

where $\left\{a_{k}\right\}$ and $\left\{b_{k}\right\}$ are constants describing the amounts of the various modes, and $\left\{\overrightarrow{d^{k}}\right\}$ are the reciprocal lattice vectors of the set $\{\vec{c} k$. If the system has a stable equilibrium, the real 
part of all the $\lambda^{(k)}$ will be negative. Therefore the first term of Eq. (2.23) represents decaying modes, whereas the second term represents growing modes. This is a generalization of the work of Paninski [1] to $N$ dimensions.

In general, a Lagrangian problem will be defined by specific starting and ending boundary conditions in time. That is, the full $N$ components of the vector $\vec{x}$ are defined at the start and finish, resulting in $2 N$ equations. The $2 N$ parameters $\left\{a_{k}\right\}$ and $\left\{b_{k}\right\}$ can then be fitted to these conditions, to give the single solution to the problem. The trajectory defined by the solution will be the one most likely to occur. However, in practice (e.g., for real neurons), we are unlikely to know the full range of boundary conditions.

\section{E. Hamilton's equations}

Before introducing a neuron model, we present the equivalent representation of the problem in the form of Hamilton's equations [9]. The Hamilton formulation uses first order differential equations in time to describe movement of a system through phase space. Formally, we define a conjugate momentum for each coordinate variable of the Lagrangian, thus doubling the dimensionality of the system but reducing it to first order in time. Unlike the Lagrangian case, which uses starting and ending conditions, the boundary conditions for the Hamiltonian approach are usually presented purely as initial conditions.

For a system with a Lagrangian given by $L=L(\dot{\vec{q}}, \vec{q}, t)$, as in Eq. (2.8) (as is conventional we now use $\vec{q}$ rather than $\vec{x}$ when using the Hamiltonian approach), we use the $N$ components of $\vec{q}$ as generalized coordinates, and for each component $q_{k}$ we define its conjugate momentum as

$$
p_{i}=\frac{\partial L(\dot{\vec{q}}, \vec{q}, t)}{\partial \dot{q}_{i}}
$$

We then construct the Hamiltonian $H$ as

$$
H(\vec{p}, \vec{q}, t)=\dot{\vec{q}}^{T} \vec{p}-L(\dot{\vec{q}}, \vec{q}, t)
$$

where we have written the $N$ conjugate momenta variables as a vector $\vec{p}$. Hamilton's equations are then given by

$$
\dot{q}_{i}=\frac{\partial H}{\partial p_{i}}, \quad \dot{p}_{i}=-\frac{\partial H}{\partial q_{i}}, \quad-\frac{\partial L}{\partial t}=\frac{\partial H}{\partial t} .
$$

For our specific case, we start with Eq. (2.8):

$$
L=\frac{1}{2}\left(\dot{q}_{k}-f_{k}\right) \Sigma_{k j}^{-2}\left(\dot{q}_{j}-f_{j}\right)
$$

where we have used $\vec{q}=\vec{x}$ (for the sake of convention) and used component form with the summation convention for repeated indices. If we assume that $\vec{f}=\vec{f}(\vec{q})$ (i.e., is not an explicit function of $\dot{\vec{q}}$ or $t$ ) we obtain our conjugate momenta as

$$
p_{i}=\frac{\partial L}{\partial \dot{q}_{i}}=\frac{1}{2}\left(\dot{q}_{k}-f_{k}\right) \Sigma_{k i}^{-2}+\frac{1}{2} \Sigma_{i j}^{-2}\left(\dot{q}_{j}-f_{j}\right)=\Sigma_{i j}^{-2}\left(\dot{q}_{j}-f_{j}\right),
$$

where the last part follows since $\Sigma^{2}$ is symmetric. Our Hamiltonian is given from Eqs. (2.25) and (2.27) as

$$
H=\dot{q}_{i} p_{i}-\frac{1}{2}\left(\dot{q}_{k}-f_{k}\right) \Sigma_{k j}^{-2}\left(\dot{q}_{j}-f_{j}\right) .
$$

To proceed, we must write $H$ in terms of $\vec{p}, \vec{q}$ (and in general $t$ ). We therefore solve for $\dot{q}_{k}$ from Eq. (2.28) and substitute into Eq. (2.29). From Eq. (2.28), we find

$$
\dot{q}_{k}=f_{k}+\Sigma_{k l}^{2} p_{l}
$$

and so substituting into Eq. (2.29) gives

$$
H(\vec{p}, \vec{q})=f_{i} p_{i}+\frac{1}{2} p_{i} \Sigma_{i l}^{2} p_{l}
$$

The Hamiltonian is now written purely as a function of the components of $\vec{q}$ and $\vec{p}$. (A $t$ variable is not required since the Lagrangian is not an explicit function of time.) From here, we can identify pseudoenergy terms. The second term on the right-hand side of Eq. (2.31), which varies as conjugate momentum squared, we identify as a pseudokinetic energy. This leaves the first term as a pseudopotential energy. In this case, since there is no explicit time dependence of the Hamiltonian, we would expect the pseudoenergy to be a conserved quantity. It is important to note, however, that $H$ is not a true "energy"- similar to $L$ it carries dimensions of inverse time.

The equations of motion for the system are found from Eq. (2.26). Substituting for $H$ from Eq. (2.31), we obtain the two equation sets

$$
\dot{q}_{i}=\frac{\partial H}{\partial p_{i}}=f_{i}+\Sigma_{i l}^{2} p_{l},
$$

which is the same as Eq. (2.30), and

$$
\dot{p}_{i}=-\frac{\partial H}{\partial q_{i}}=-\frac{\partial f_{k}}{\partial q_{i}} p_{k} .
$$

Note that there are $2 \mathrm{~N}$ equations in total. The Euler-Lagrange equations (2.10) can be obtained by differentiating Eq. (2.32) with respect to time, substituting for $\dot{p}_{i}$ from Eq. (2.33), and resubstituting for $p_{k}$ from Eq. (2.32). Hamilton's equations (2.32) and (2.33) are equivalent to the Euler-Lagrange equations (2.10), and are a general result. This form is not, however, necessarily more useful than the Lagrangian approach, but, as explained in Sec. II F below, it leads naturally to a physical interpretation of the changes in the system on the approach to an action potential.

\section{F. Interpretation}

We find that the conjugate momentum $\vec{p}$ has a physical interpretation. Comparing Eq. (2.32) with the original equation of motion (2.1), we see that (in matrix form) 


$$
\Sigma^{2} \vec{p}=\vec{v}
$$

i.e., the conjugate momentum $\vec{p}$ is simply $\Sigma^{-2} \vec{v}$. This is nonintuitive since $\vec{v}$ represents the noise process, yet the Hamilton equation for the time derivative of $\vec{p}$ is well defined. These facts are reconciled when we recall that the Lagrangian and Hamiltonian approaches represent the most likely path of the system; so the relationship between $\vec{p}$ and $\vec{v}$ can be viewed as a statement of what the most-likely noise input $\vec{v}$ is for given starting and ending conditions. We will look at this result in more detail as part of the numerical simulations described below.

In the case of small fluctuations about the equilibrium point (which by construction is at $\vec{q}=0$ ), we can define $\vec{f}(\vec{q})=M \vec{q}$, or, in components, $f_{k}=M_{k j} q_{j}$, and so Eq. (2.33) becomes

$$
\dot{p}_{i}=-M_{k i} p_{k}=-M_{i k}^{T} p_{k}
$$

Recall that, for a stable equilibrium, $M$ is a negative definite matrix, so the real parts of its eigenvalues are negative. Clearly if $\vec{p}$ is initially an eigenvector of $M^{T}$, it will remain as an eigenvector and so grow exponentially in time according to the corresponding eigenvalue. Therefore, we can consider the growing conjugate momentum as giving rise to the growing modes of Eq. (2.23). For small fluctuations $\vec{f}(\vec{q})=M \vec{q}$ where $M$ is a constant matrix, and so the time constant for the growth in $\vec{p}$ depends upon the eigenvalues of $M$, rather than the size of the noise $\Sigma$-i.e., it depends on the closeness to the critical driving current. However, for larger deviations this no longer applies and the extent of the noise $\Sigma$, which influences $\vec{q}$ through Eq. (2.32), will now influence $\vec{p}$ through Eq. (2.33). Very close to firing we expect the noise terms to be more dominant; for example, Badel et al. found that close to the threshold of a two-component integrate and fire neuron the averaged trajectory of the membrane potential depends on the noise term in addition to the neural time constants [3].

\section{SINGLE NEURON MODEL}

\section{A. Model formulation}

To illustrate the Hamiltonian approach, we wish to select a model of a neuron that is both mathematically simple and physiologically reasonable. For this reason we choose the model due to Wilson [8]. This model was designed to encompass the physiological accuracy of a Hodgkin-Huxley neuron [13] (in an approximate two-component form in the manner of Rinzel [14]) while retaining the mathematical simplicity provided by the simple spiking equations of FitzHugh [15] and Nagumo [16]. The equations are capable of describing both integrator and resonator type neurons, with simple changes to the form of the equations. In this work, we focus on an integrator neuron, such as a cortical neuron.

Wilson uses just two coupled differential equations in time, the first to describe the membrane potential, the second to describe a generalized recovery process. To these equations we add zero-mean white-noise inputs, in the manner of Steyn-Ross et al. [7]. The equations of the model are written
TABLE I. The parameters and constants for the model of Wilson, for the cortical neuron. The reader should, however, note the following. (i) The equation for $G(R)$ quoted by Wilson in Ref. [8] is incorrect; the quadratic component has been written as $0.33 \mathrm{dV}^{-2}$ but should read $3.3 \mathrm{dV}^{-2}$. This mistake is acknowledged by Wilson in Ref. [19]. (ii) In Ref. [7] the full precision defined for $\gamma$ by Wilson, namely, $\gamma=1.26652$, has been used; however, it has been quoted in Table I of that reference as $\gamma=1.267$. (iii) In this paper we have used the reduced precision form of $\gamma$, i.e., 1.267. This means that our critical current and associated time scales do not quite agree with those of Ref. [7].

\begin{tabular}{lcc}
\hline \hline Symbol & Value & Unit \\
\hline$C$ & 1.0 & $\mu \mathrm{F} \mathrm{cm}^{-2}$ \\
$\tau_{R}$ & 5.6 & $\mathrm{~ms}$ \\
$E_{\mathrm{Na}}$ & 48 & $\mathrm{mV}$ \\
$E_{\mathrm{K}}$ & -95 & $\mathrm{mV}$ \\
$g_{\mathrm{K}}$ & 26 & $\mathrm{mS} \mathrm{cm}^{-2}$ \\
$a$ & $33.8 \times 10^{-4}$ & $\mathrm{mS} \mathrm{cm}^{-2} \mathrm{mV}^{-2}$ \\
$b$ & $47.58 \times 10^{-2}$ & $\mathrm{mS} \mathrm{cm}^{-2} \mathrm{mV}^{-1}$ \\
$c$ & 17.81 & $\mathrm{mS} \mathrm{cm}^{-2}$ \\
$\alpha$ & $3.30 \times 10^{-4}$ & $\mathrm{mV}^{-2}$ \\
$\beta$ & $3.798 \times 10^{-2}$ & $\mathrm{mV}^{-1}$ \\
$\gamma$ & 1.267 & $\mathrm{dimensionless}^{-2}$ \\
$\sigma_{1}$ & parameter & $\mu \mathrm{A} \mathrm{cm}^{-2} \mathrm{~ms}^{1 / 2}$ \\
$\sigma_{2}$ & parameter & $\mathrm{ms} \mathrm{s}^{1 / 2}$ \\
\hline \hline
\end{tabular}

$$
C \frac{d V}{d t}=-g_{\mathrm{Na}}(V)\left(V-E_{\mathrm{Na}}\right)-g_{\mathrm{K}} R\left(V-E_{\mathrm{K}}\right)+I_{\mathrm{dc}}+\sigma_{1} \xi_{1}(t),
$$

$$
\tau_{R} \frac{d R}{d t}=-R+G(V)+\sigma_{2} \xi_{2}(t)
$$

where $V$ is the membrane potential, and $R$ is a dimensionless "recovery" variable. The parameters for the model are the membrane capacitance per unit area $C$; the sodium and potassium reversal potentials $E_{\mathrm{Na}}$ and $E_{\mathrm{K}}$, respectively; the potassium channel conductance per unit area $g_{\mathrm{K}}$; the direct component of the driving current $I_{\mathrm{dc}}$; scaling of the noise $\sigma_{1}$ and $\sigma_{2}$; and a time-constant for the recovery process $\tau_{R}$. The terms $\xi_{1}$ and $\xi_{2}$ are uncorrelated white-noise inputs, with properties

$$
\left\langle\xi_{i}(t)\right\rangle=0, \quad\left\langle\xi_{i}(t) \xi_{j}\left(t^{\prime}\right)\right\rangle=\delta_{i j} \delta\left(t-t^{\prime}\right) .
$$

Note that in this continuous form, the noise terms, unlike those of the discrete time equation (2.2), are dimensioned; they carry dimensions of inverse square-root time. For a cortical neuron, the functions $g_{\mathrm{Na}}(V)$ and $G(V)$ are given by second-order polynomials $g_{\mathrm{Na}}(V)=a V^{2}+b V+c$ and $G(V)$ $=\alpha V^{2}+\beta V+\gamma$. The values of the parameters and constants are given in Table I.

For this paper, the driving current $I_{\mathrm{dc}}$ and the noise strengths $\sigma_{1}$ and $\sigma_{2}$ will be varied. Equation (3.1) is written in terms of currents, and Eq. (3.2) in similar form; to trans- 
form these to the form of Eq. (2.1), we divide Eq. (3.1) by $C$ and Eq. (3.2) by $\tau_{R}$.

For $I_{\mathrm{dc}}$ above the threshold of approximately $21.809 \mu \mathrm{A} \mathrm{cm}^{-2}$ the neuron enters a limit cycle, firing spikes at regular intervals, the spike-rate increasing smoothly as $I_{\mathrm{dc}}$ is raised. For a driving current below threshold, there is a stable node solution to the equations, and we expect small fluctuations in $V$ and $R$ around their equilibrium values. However, the noise may be large enough for spikes to be produced. In Ref. [7], Steyn-Ross et al. analyzed the dynamics of the model as $I_{\mathrm{dc}}$ was increased towards threshold. The closer the driving current is to threshold, the more frequently spikes will be produced for a given noise level; and an increase in noise will increase the spike rate.

We now rewrite the model equations (3.1) and (3.2) in terms of the Hamiltonian formalism. First, we define our state vector $\vec{q}$ as a two-component vector consisting of the membrane potential $V$ and recovery variable $R$; i.e., $q_{1}=V$, $q_{2}=R$. The components of the function $\vec{f}$ then follow directly from Eqs. (3.1) and (3.2):

$$
\begin{gathered}
f_{1}=\frac{1}{C}\left[-g_{\mathrm{Na}}\left(q_{1}\right)\left(q_{1}-E_{\mathrm{Na}}\right)-g_{\mathrm{K}} q_{2}\left(q_{1}-E_{\mathrm{K}}\right)+I_{\mathrm{dc}}\right], \\
f_{2}=\frac{1}{\tau_{R}}\left[-q_{2}+G\left(q_{1}\right)\right] ;
\end{gathered}
$$

and the noise terms are simply $\Sigma_{11}=\sigma_{1} / C, \Sigma_{22}=\sigma_{2} / \tau_{R}$, and $\Sigma_{12}=\Sigma_{21}=0$. Hamilton's equations (2.32) and (2.33) give us four coupled first-order differential equations

$$
\begin{gathered}
\dot{q}_{1}=\left(\frac{\sigma_{1}}{C}\right)^{2} p_{1}+\frac{1}{C}\left[-\left(a q_{1}^{2}+b q_{1}+c\right)\left(q_{1}-E_{\mathrm{Na}}\right)\right. \\
\left.-g_{\mathrm{K}} q_{2}\left(q_{1}-E_{\mathrm{K}}\right)+I_{\mathrm{dc}}\right] \\
\dot{q}_{2}=\left(\frac{\sigma_{2}}{\tau_{R}}\right)^{2} p_{2}+\frac{1}{\tau_{R}}\left(-q_{2}+\alpha q_{1}^{2}+\beta q_{1}+\gamma\right) \\
\dot{p}_{1}=\frac{-1}{C}\left[-3 a q_{1}^{2}+\left(2 a E_{\mathrm{Na}}-2 b\right) q_{1}-g_{\mathrm{K}} q_{2}+\left(b E_{\mathrm{Na}}-c\right)\right] p_{1} \\
-\frac{1}{\tau_{R}}\left(2 \alpha q_{1}+\beta\right) p_{2}, \\
\dot{p}_{2}=\frac{1}{C}\left[g_{\mathrm{K}}\left(q_{1}-E_{\mathrm{K}}\right)\right] p_{1}+\frac{1}{\tau_{R}} p_{2} .
\end{gathered}
$$

Note that $p_{1}$ has dimensions of inverse voltage, and $q_{2}$ $=R$ and $p_{2}$ are both dimensionless. Equations (3.8) and (3.9) can be written in matrix form as

$$
\dot{\vec{p}}=A(\vec{q}) \vec{p}
$$

Close to equilibrium, the matrix $A$ is constant (independent of $\vec{q}$ ), and equal to minus the $M^{T}$ of Eq. (2.35), but away from equilibrium this is no longer true. In particular, on the approach to an action potential, the nonlinear part of the dynamics begins to dominate the restoring dynamics represented by the linear region [7].

From the Hamiltonian of Eq. (2.31), we identify the pseudopotential energy $H_{V}$ as

$$
\begin{aligned}
H_{V}= & \vec{f} \cdot \vec{p}=\frac{1}{C}\left[-g_{\mathrm{Na}}\left(q_{1}\right)\left(q_{1}-E_{\mathrm{Na}}\right)-g_{\mathrm{K}} q_{2}\left(q_{1}-E_{\mathrm{K}}\right)+I_{\mathrm{dc}}\right] p_{1} \\
& +\frac{1}{\tau_{R}}\left[-q_{2}+G\left(q_{1}\right)\right] p_{2}
\end{aligned}
$$

and the pseudokinetic energy as

$$
H_{T}=\frac{1}{2} \vec{p}^{T} \Sigma^{2} \vec{p}=\frac{p_{1}^{2} \sigma_{1}^{2}}{2 C^{2}}+\frac{p_{2}^{2} \sigma_{2}^{2}}{2 \tau_{R}^{2}} .
$$

The matrix $\Sigma^{2}$ is now recognizable as an inverse inertia. For example, in the limit of $\Sigma \rightarrow 0$ (i.e., vanishingly small noise) the system would not depart from its equilibrium position, consistent with it having a massive inertia.

\section{B. Implementation}

The equations (3.6)-(3.9) have been coded with the Matlab programming language. For a set of initial conditions (where $q_{1}, q_{2}, p_{1}$, and $p_{2}$ are defined at $t=0$ ) the variables can be iterated forward in time. For most values of the input parameters $I_{\mathrm{dc}}, \sigma_{1}$ and $\sigma_{2}$, the model eventually "fires" an action potential; $q_{1}(=V)$ rises very steeply while $q_{2}(=R)$ drops. The numerics then break down as $p_{1}$ and $p_{2}$ rapidly get very large.

What does the trajectory tell us? Since Hamilton's equations are equivalent to the Lagrangian approach, the trajectory will be the most likely path taken for the neuron between its starting point and any given point on the trajectory. We can therefore use this method to define the most likely trajectory of the variables $V$ and $R$ on the approach to an action potential.

Simple iteration of equations (3.6)-(3.9) forward in time is, however, not practical. The reason for this is the rapidly increasing values of $p_{1}$ and $p_{2}$. For a system close to equilibrium, Eq. (2.35) applies, and we see that the conjugate momenta will grow rapidly. Moreover, the most rapid growth will be due to the eigenvalue of the matrix $M$ that is most negative. In Ref. [7], Steyn-Ross et al. have analyzed the eigenvalues of the Wilson model, showing that, for a system close to threshold, there is one "slow" and one "fast" eigenmode. The fast eigenmode has a large, negative eigenvalue and therefore dominates the behavior of the conjugate momenta in the limit Hamiltonian approach. In practice, this means that iterating the Hamiltonian equations forwards in time leads to an almost immediate firing of a "spike," during which the numerics breaks down.

To avoid this problem, we remove the fast eigenvalue, by projecting the vector $\vec{p}$ onto its slow eigenvalue, at every time step. Mathematically, we find the normalized eigenvectors of the matrix $A(\vec{q})$ of Eq. (3.10), given by $\vec{p}_{s}$ and $\vec{p}_{f}$, where the subscripts $s$ and $f$ denote slow and fast, respectively. We then write $\vec{p}$ in terms of the normalized fast and slow eigenvalues: 

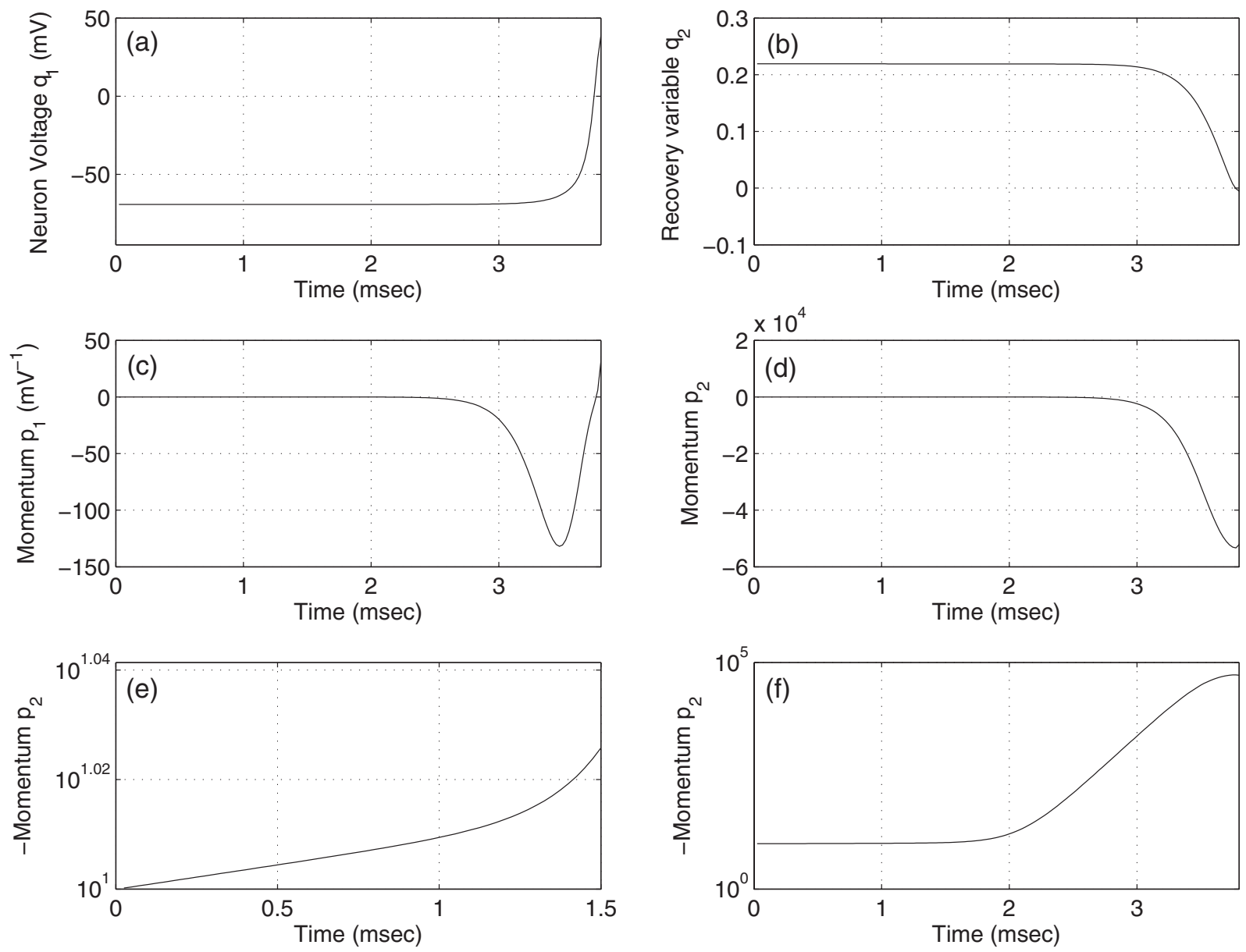

FIG. 1. A plot of the full "fast" solution to Hamilton's equations for the Wilson neuron for the case of $I_{\mathrm{dc}}=21.475 \mu \mathrm{A} \mathrm{cm}{ }^{-2}, \sigma_{1}$ $=0.02 \mu \mathrm{A} \mathrm{cm}^{-2} \mathrm{~ms}^{1 / 2}$, and $\sigma_{2}=0.02 \mathrm{~ms}^{1 / 2}$. Parts (a)-(d) show the variables $q_{1}=V, q_{2}=R, p_{1}$, and $p_{2}$, respectively, as functions of time. Part (e) shows the growth in $-p_{2}$ at small times on a log scale; part (f) shows the growth closer to the firing event. Note how the two time scales are very different, corresponding to the slow (e) and fast (f) scales.

$$
\vec{p}=k_{1} \vec{p}_{s}+k_{2} \vec{p}_{f}
$$

where $k_{1}$ and $k_{2}$ are scalars, and simply remove the fast term, i.e.,

$$
\vec{p} \rightarrow k_{1} \vec{p}_{s}
$$

This approximation would be precise if we are always close to equilibrium, i.e., the matrix $A(\vec{q})=M^{T}$ does not change with time, and so its eigenvectors are constant. Then a vector $\vec{p}$ that is parallel with $\vec{p}_{s}$ would be expected to remain parallel, for all times.

We therefore verify the Hamiltonian approach in Sec. IV below with the following method. We choose to start the system at equilibrium (i.e., $q_{1}=V$ and $q_{2}=R$ are chosen to be their equilibrium values, so that, in the absence of noise $\left(\sigma_{1}, \sigma_{2} \rightarrow 0\right)$, both $\dot{q}_{1}$ and $\dot{q}_{2}$ would be zero). We also select initial values of the conjugate momenta $p_{1}$ and $p_{2}$. We then iterate the equations (3.6)-(3.9) forward in time with a second-order Runge-Kutta method, using a time step of $5 \mu \mathrm{s}$, projecting out the fast eigenvalue at every time step with the method of Eq. (3.14). This results in a "spike" being generated. Typically, the numerics breaks down after the spike. At the time at which the spike forms (which we sig- nify by when $V$ crosses an arbitrary threshold, e.g., $-55 \mathrm{mV}$ ), we record the values of $q_{1}$ and $q_{2}$, which we denote by $V_{T}$ and $R_{T}$, respectively, and time taken, $T$. We then have an "end value" situation: the path indicates the "most-likely" trajectory for the system to travel from its equilibrium position to the position $V_{T}$ and $R_{T}$ in exactly a time $T$ (subject to the validity of the slow approximation described above).

Finally, we can simulate the equations of Wilson, Eqs. (3.1) and (3.2) directly. (Note these equations include noise input.) We do this with a second-order predictor-corrector method [17]. We have used the same time step of $5 \mu \mathrm{s}$. We start the system at equilibrium and run for a time $T$. After this time, we look at the values of $V$ and $R$, and "accept" the run if they are sufficiently close to $V_{T}$ and $R_{T}$. This means that the simulation has produced a path with the correct starting and finishing points. We then run the simulation again, until we have collected of order hundred or more acceptable sequences. We then average these sequences over time to produce an "average" neuron trajectory, and compare it to the Hamiltonian simulation. However, success rates for acceptable neuron simulations are often very low (less than $1 \%$ ).

Note that when we do not project onto the slow eigenvector, as in Eq. (3.14), we find that not only does the neuron 


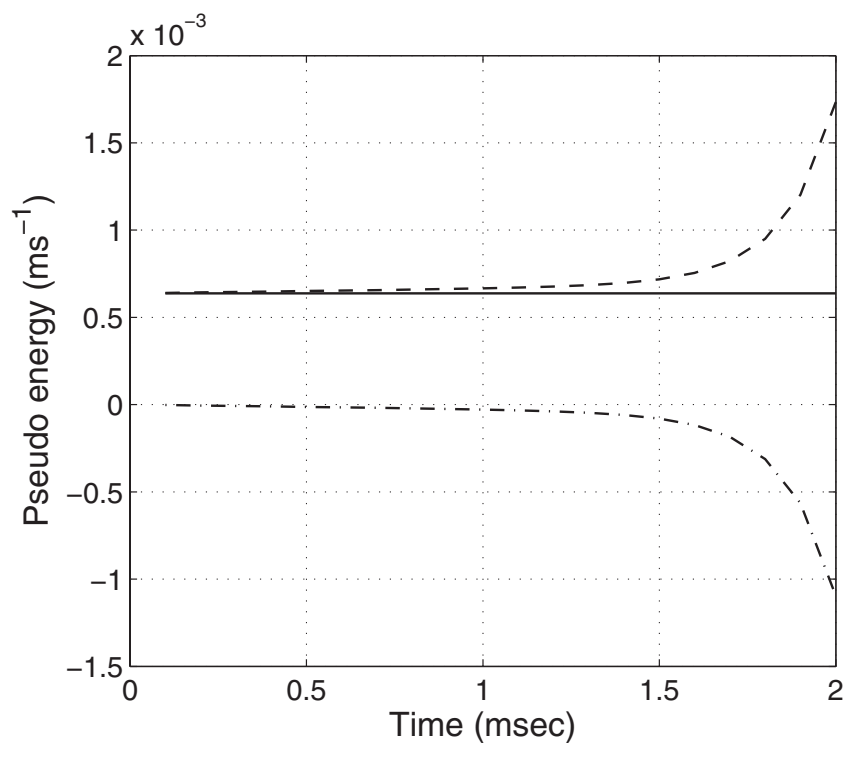

FIG. 2. A plot of the pseudokinetic (dash) and pseudopotential (dot-dash) energies as a function of time, for the simulation of Fig. 1. The sum of the two (solid line) is conserved.

fire very quickly, but the end points $V_{T}$ and $R_{T}$ are such that there is an extremely low success rate in terms of simulations-i.e., only a tiny number of simulated runs end with $V$ and $R$ values close to the target $V_{T}$ and $R_{T}$. This makes a comparison of the full Hamiltonian method with the simulations impractical. In theory, one would expect there to exist initial values of $p_{1}$ and $p_{2}$ such that any start and finish points could be reproduced; however, we have no way of knowing what those starting points might be.

\section{RESULTS AND DISCUSSION}

\section{A. Full Hamiltonian simulations}

First, we present results for the full Hamiltonian method, with no projection onto the slow eigenvalue. Figure 1 corresponds to the case of $I_{\mathrm{dc}}=21.475 \mu \mathrm{A} \mathrm{cm}^{-2}$ (i.e., close to threshold), and $\sigma_{1}=0.02 \mu \mathrm{A} \mathrm{cm}^{-2} \mathrm{~ms}^{1 / 2}, \sigma_{2}=0.02 \mathrm{~ms}^{1 / 2}$. In the four plots (a)-(d) we show the variation of the variables $q_{1}, q_{2}, p_{1}$, and $p_{2}$, respectively, as functions of time, up to the onset of the firing event (spike). The initial values of $p_{1}$ and $p_{2}$ were chosen to lie along a slow eigenvalue. The onset of the spike is extremely rapid, but is signalled by the rapid growth in the magnitude of $p_{1}$ and $p_{2}$. In parts (e) and (f) we focus on the exponential growth behavior of $p_{2}$ by plotting it on a $\log$ scale. (The negative of $p_{2}$ is taken to ensure the logarithm is real.) The two separate time-scales are clearly seen; part (e) shows the case for small times where $p_{2}$ grows according to the slow-time scale (since the initial conditions were set as the slow eigenvector), whereas part (f) shows later times where the fast eigenvalue becomes important.

Since the Hamiltonian is not an explicit function of time, it should be a conserved quantity. This is demonstrated by a plot of the pseudoenergy terms in Fig. 2. The kinetic part increases on the approach to the spike; the potential part, which starts near zero, decreases, becoming negative. Overall, the sum of the two is constant.
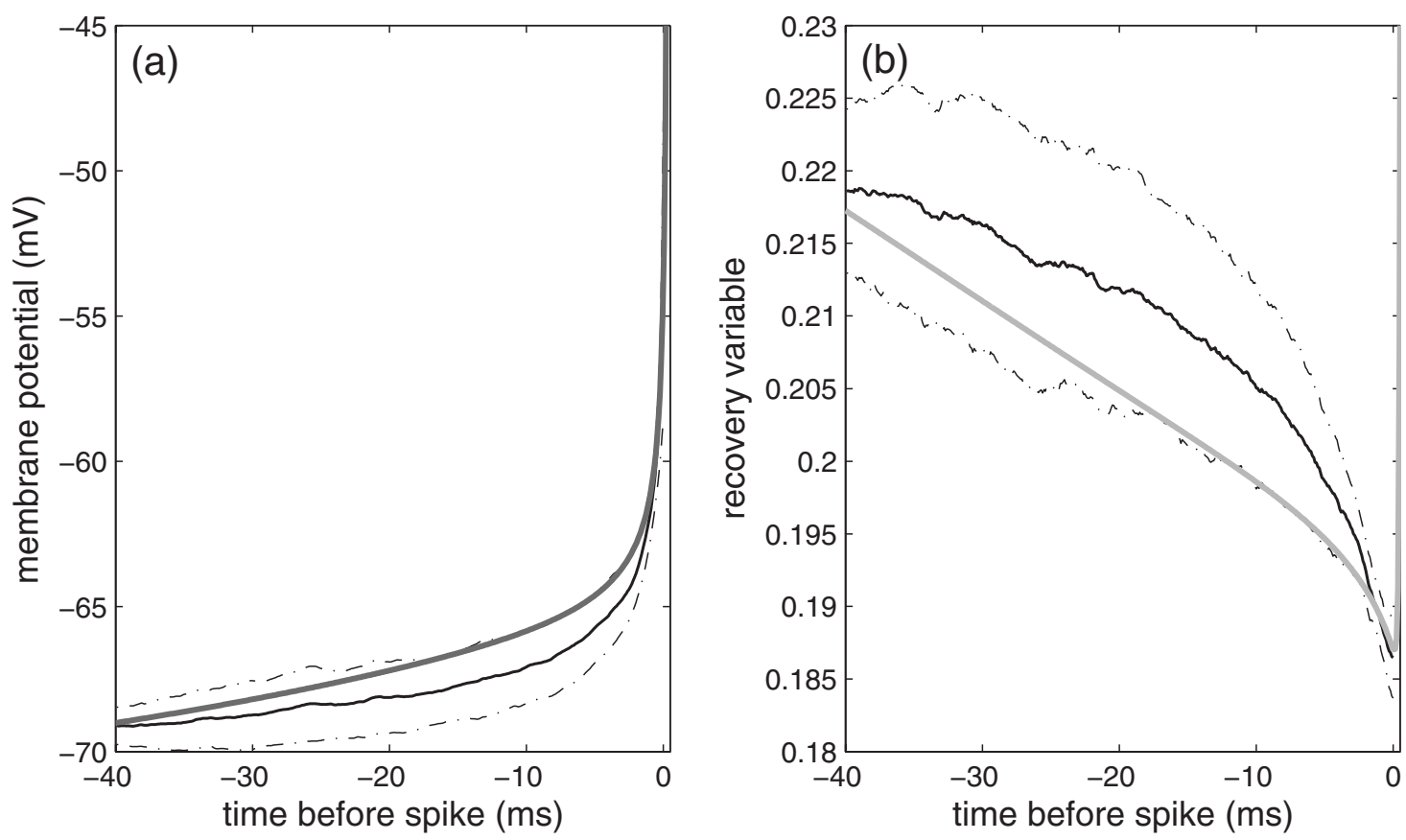

FIG. 3. A comparison of the simulated Wilson neuron and the Hamiltonian approach, for the case of $I_{\mathrm{dc}}=21.475 \mu \mathrm{A} \mathrm{cm}{ }^{-2}, \sigma_{1}$ $=0.02 \mu \mathrm{A} \mathrm{cm}^{-2} \mathrm{~ms}^{1 / 2}$, and $\sigma_{2}=0.02 \mathrm{~ms}^{1 / 2}$. The Hamiltonian solution using the "slow" approximation is shown by the thick line; the mean result of the 119 acceptable simulations out of 10000 trials is shown by the thinner line. The spread in the simulated trajectories is indicated by the dotted lines which are placed one standard deviation above and below the mean trajectory. Part (a) shows the solution for the membrane potential $V$; part (b) shows the solution for the recovery variable $R$. The sequencies have been aligned so that they cross $-55 \mathrm{mV}$ at $t=0 \mathrm{~ms}$, therefore the time sequences are not all exactly $43.2 \mathrm{~ms}$ long. 


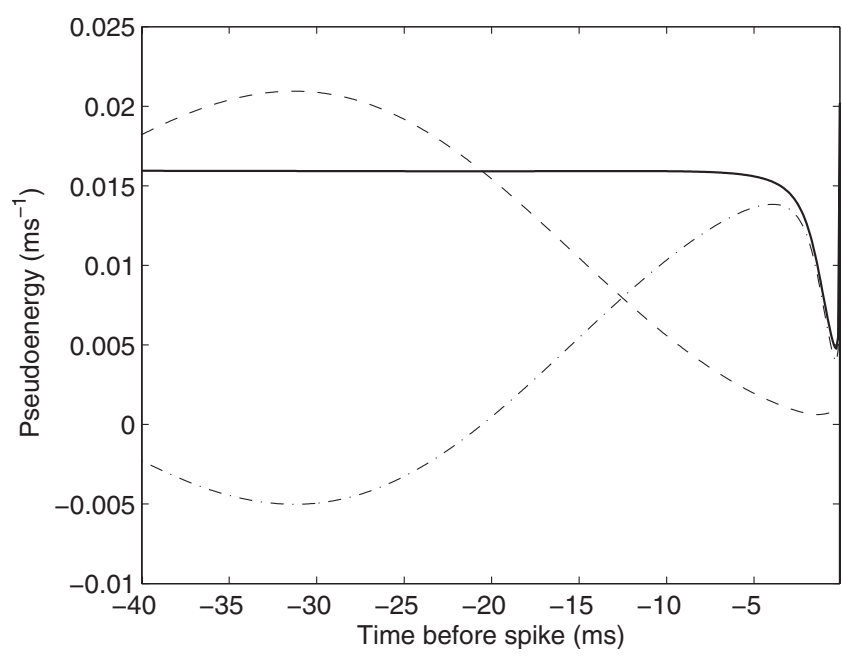

FIG. 4. A plot of the pseudokinetic (dash) and pseudopotential (dot-dash) energies as a function of the time before a spike, for the Hamiltonian simulation of Fig. 3 with a slow approximation. The total pseudoenergy (solid line) is conserved at early times but not in the final $5 \mathrm{~ms}$ before the firing event.

\section{B. Spiking events}

We continue by presenting a comparison between trajectories produced by the Hamiltonian approach of Eqs. (3.6)-(3.9) and direct simulation of the Wilson model, (3.1) and (3.2). In Fig. 3 we show the case for the conditions of Fig. 1, namely, $I_{\mathrm{dc}}=21.475 \mu \mathrm{A} \mathrm{cm}^{-2}, \quad \sigma_{1}$ $=0.02 \mu \mathrm{A} \mathrm{cm}^{-2} \mathrm{~ms}^{1 / 2}$, and $\sigma_{2}=0.02 \mathrm{~ms}^{1 / 2}$. The initial values of $p_{1}$ and $p_{2}$ were chosen to be parallel to the slow eigen-

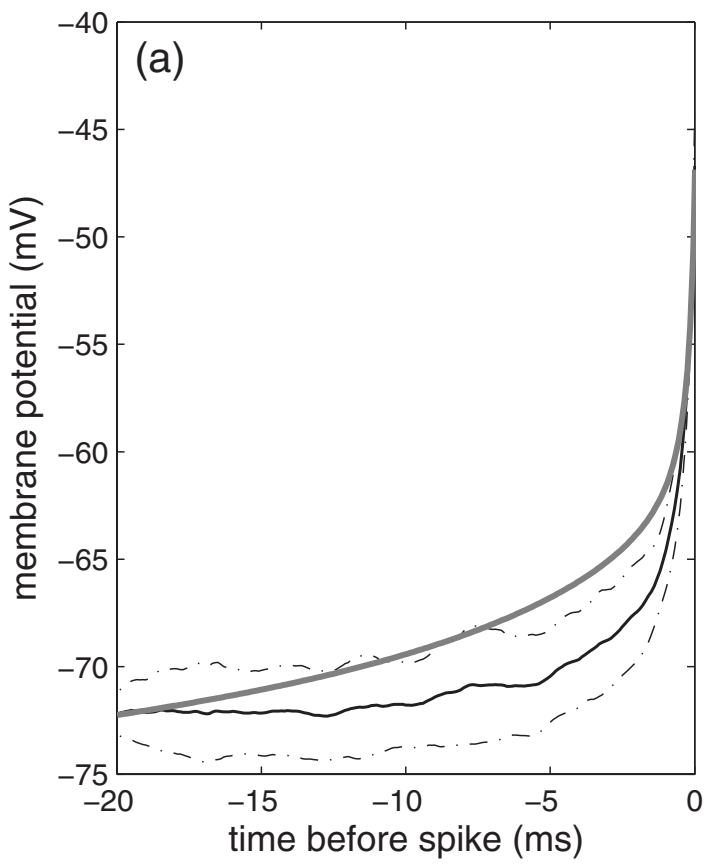

vector' and their magnitude chosen to produce a spike after a reasonable interval of time, in this case about $40 \mathrm{~ms}$. The end point of the Hamiltonian simulation was considered to be $T=43.2 \mathrm{~ms}$ where $q_{1}=V$ crossed $-55 \mathrm{mV}$; at this point $q_{2}$ $=R$ reached 0.19 . At the end point, the membrane potential was rising very rapidly, whereas the recovery variable was fairly constant. For this reason, tolerances were chosen on the end points as follows: for $V_{T},-60 \mathrm{mV}$ to $+20 \mathrm{mV}$; for $R_{T}, 0.18$ to 0.21 . Simulations of the Wilson model were then performed over this time period, and accepted if the end points for $V$ and $R$ were within these tolerances (and no previous spike was evident in the time period). Note that both $V$ and $R$ needed to be within these bounds for the run to be accepted as having the correct finishing point.

Out of a total of 21000 simulations, only 119 trajectories reproduced the correct $V_{T}$ and $R_{T}$ end values after $43.2 \mathrm{~ms}$. In Fig. 3 we display the simulated trajectories in the form of the mean trajectory for both $V$ and $R$, and the standard deviation in the trajectory. When plotting these, we align the trajectories precisely in time so they cross the chosen threshold $(-55 \mathrm{mV})$ at the same point in time. We also show the Hamiltonian solution (using the slow approximation). We observe that the Hamiltonian approach is a reasonable approximation to the simulations, but it is about one standard deviation high in $V$ for a significant part of the trajectory, and one standard deviation low in $R$.

The behavior of the pseudoenergy terms for the Hamiltonian solution is shown in Fig. 4. In this case the Hamiltonian is conserved for small times, but the total pseudoenergy falls as the spike is approached. This loss in energy may be attributable to the effects of the slow approximation. Interestingly, in this case the kinetic and potential pseudoener-

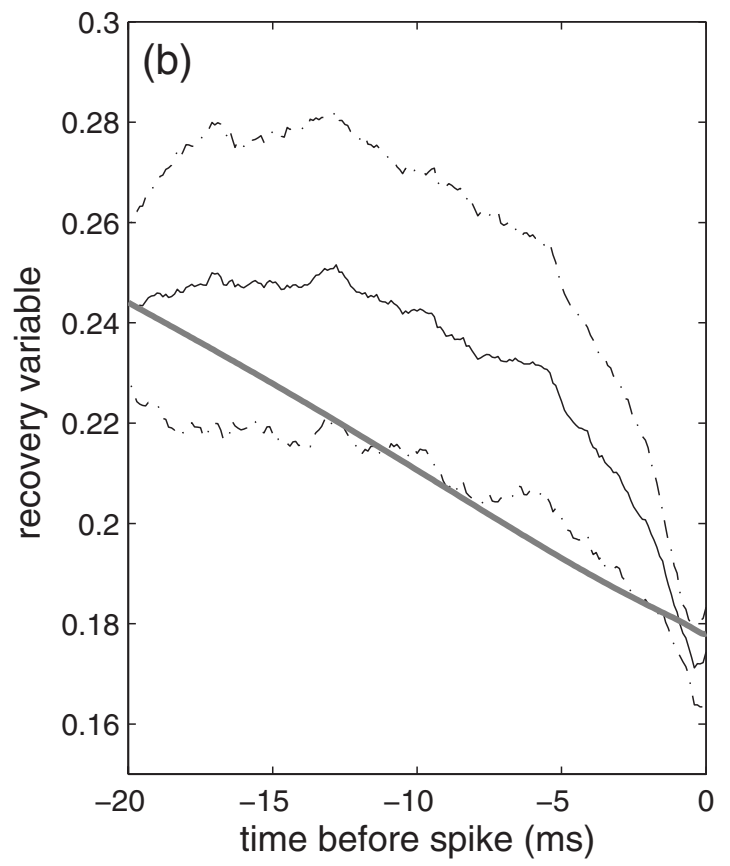

FIG. 5. A comparison of the simulated Wilson neuron and the slow Hamiltonian approach, for the case of $I_{\mathrm{dc}}=15.0 \mu \mathrm{A} \mathrm{cm}{ }^{-2}, \sigma_{1}$ $=0.1 \mu \mathrm{A} \mathrm{cm}^{-2} \mathrm{~ms}^{1 / 2}$, and $\sigma_{2}=0.1 \mathrm{~ms}^{1 / 2}$. There were 80 sequences accepted from 36000 trials. The Hamiltonian solution is shown by the thick line; the mean result of the simulations by the thinner line. The spread in the simulated trajectories is indicated by the dotted lines which are placed one standard deviation above and below the mean trajectory. Part (a) shows the solution for the membrane potential $V$; part (b) shows the solution for the recovery variable $R$. 

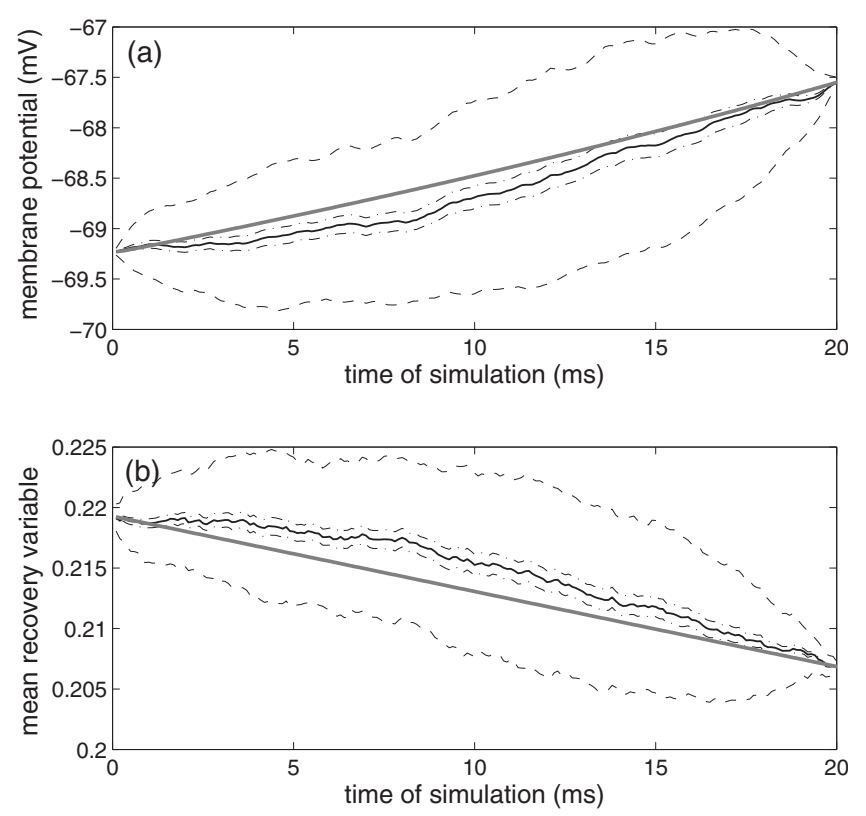

FIG. 6. A comparison of the simulated Wilson neuron and the slow Hamiltonian approach, for the case of $I_{\mathrm{dc}}=21.475 \mu \mathrm{A} \mathrm{cm}^{-2}$, $\sigma_{1}=0.02 \mu \mathrm{A} \mathrm{cm}^{-2} \mathrm{~ms}^{1 / 2}$, and $\sigma_{2}=0.02 \mathrm{~ms}^{1 / 2}$. The Hamiltonian solution is shown by the thick gray line; the mean result of the 75 accepted simulations out of 10000 attempts is shown by the thinner solid line. The spread in the simulated trajectories is indicated by the dashed lines which are placed one standard deviation above and below the mean trajectory. Also shown by the dot-dash lines are the standard uncertainty in the mean (i.e., the standard deviation divided by $\sqrt{75-1}$, where 75 is the number of accepted simulations); these lines are one standard uncertainty above and below the mean, respectively. Part (a) shows the solution for the membrane potential $V$; part (b) shows the solution for the recovery variable $R$.

gies reach turning points about $30 \mathrm{~ms}$ before the firing event. We have not investigated this phenomenon further.

We also show a similar comparison for a lower driving current $I_{\mathrm{dc}}=15.0 \mu \mathrm{A} \mathrm{cm}^{-2}$ in Fig. 5. In order to produce spiking events, we have needed to increase the noise level, in this case by a factor of 5 . The membrane potential $V$ reached $-55 \mathrm{mV}$ after $21.3 \mathrm{~ms}$. The thresholds for $V_{T}$ and $R_{T}$ after $21.3 \mathrm{~ms}$ were chosen as for $V_{T},-50 \mathrm{mV}$ to $+20 \mathrm{mV}$; for $R_{T}$, 0.18 to 0.22 . In this case there were only 80 acceptable simulated sequences from a total of 36000 simulations. Results are broadly similar to those of Fig. 3 but less good; the membrane potential in the Hamiltonian approach is somewhat high, whereas the recovery variable is somewhat low. In this case of a system well-below threshold we believe that the slow approximation is less valid-in the simulations the onset of the spike is fairly rapid, due to the high noise required to cause a firing event, and we would expect the fast eigenvalue of the Hamiltonian system to play some part in this. Conversely, close to threshold and in the limit of small noise the dynamics of the system is dominated by the slow time scale [7] and the slow approximation is more reasonable.

\section{Validity of the slow approximation}

In the above plots, we have focused on the case of a spike event, but this need not be the case. During an action poten- tial, we would certainly expect the slow-approximation to break down, because the matrix $A(\vec{q})$ of Eq. (3.10) is not constant, meaning that a vector $\vec{p}$ that initially lies along an eigenvector of $A$ does not at future times. However, if we only consider situations close to equilibrium, $A$ is approximately constant, equal to $-M^{T}$ of Eq. (2.35), and therefore a vector $\vec{p}$ that initially lies along the slow eigenvector is expected to do so for all times. In Fig. 6 we show a comparison of the Hamiltonian and simulation approaches for the case of a small deviation from equilibrium. Again, we have chosen the close-to-threshold situation of $I_{\mathrm{dc}}=21.475 \mu \mathrm{A} \mathrm{cm}^{-2}, \sigma_{1}$ $=0.02 \mu \mathrm{A} \mathrm{cm}^{-2} \mathrm{~ms}^{1 / 2}$, and $\sigma_{2}=0.02 \mathrm{~ms}^{1 / 2}$. In this case the end point of the Hamiltonian approach is chosen to be well before a spike forms. The acceptable range of simulation end points after a time $T$ of $20.0 \mathrm{~ms}$ were strongly confined: for $V_{T},-67.80 \mathrm{mV}$ to $-67.29 \mathrm{mV}$; for $R_{T}, 0.2053$ to 0.2084 . This led to a low acceptance rate; just 75 simulated sequences were accepted out of a total of 10000 attempts. Figure 6 shows that the Hamiltonian approach now performs much better, and tracks the mean membrane potential and recovery variable of the simulations well.

\section{Significance of the noise on spike formation}

Equation (2.34) indicates that the conjugate momentum vector $\vec{p}$ is directly related to the most likely noise input to the stochastic system. Given the exponential growth of $\vec{p}$, we infer that, on the approach to a spike, a simulated neuron would show an exponential change in its noise inputs. This is a surprising prediction-one would expect on average the noise inputs to be zero, but the implication is that it is the change in noise input that causes the spike event. Indeed, experimentally Rudolph et al. have shown that a spike is generated by a drop in inhibition that becomes more severe as the firing event is approached [6]. Also, Pospischil et al. [5] have already shown this shift in conductance with a numerical model, in their case a three-component model of Destexhe et al. [18] with conduction noise, and in their theoretical and numerical analyses Badel et al. suggest inhibitory fluctuations are at least as important as those of excitation [3]. The Hamiltonian approach presents a clear explanation on a physical basis of why this should bespecifically it is the increasing negative bias in the recovery conjugate momentum (i.e., noise) that triggers an action potential.

To demonstrate this nonintuitive exponential change in noise input predicted from the growth of $\vec{p}$ (see Fig. 1), we analyze the random number sequences for simulations of the Wilson model that occur in the build-up to a spike. In Fig. 7 we plot the mean noise against time for the two noise inputs to the simulations of Eqs. (3.1) and (3.2), for $I_{\mathrm{dc}}=21.4 \mu \mathrm{A} \mathrm{cm}^{-2}, \quad \sigma_{1}=0.03 \mu \mathrm{A} \mathrm{cm}^{-2} \mathrm{~ms}^{1 / 2}$, and $\sigma_{2}$ $=0.03 \mathrm{~ms}^{1 / 2}$. We do this by undertaking many simulations (500 for the case of the figure), selecting those that produce action potentials within a given time frame (in this case 15-60 ms), aligning them in time at the instant that the membrane potential crosses a certain threshold, analyzing the random number sequences in the period of time leading up to the spike. We average these random numbers over short 

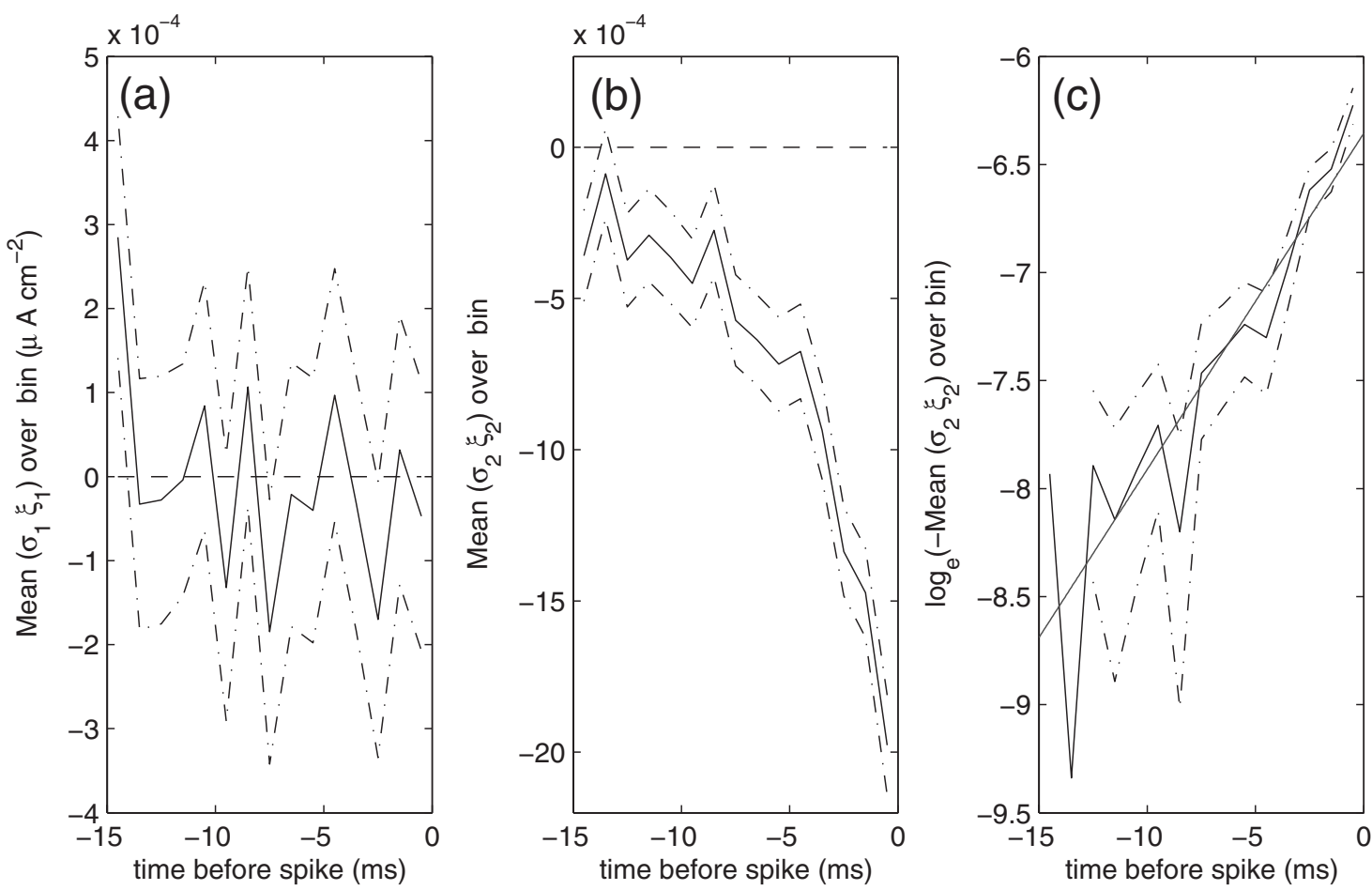

FIG. 7. A plot of the mean noise input to the Wilson model over the $15 \mathrm{~ms}$ preceding a spike, for the case of $I_{\mathrm{dc}}=21.4 \mu \mathrm{A} \mathrm{cm}{ }^{-2}, \sigma_{1}$ $=0.03 \mu \mathrm{A} \mathrm{cm} \mathrm{cm}^{-2} \mathrm{~ms}^{1 / 2}$, and $\sigma_{2}=0.03 \mathrm{~ms}^{1 / 2}$. A total of 190 simulations were accepted from 500 trials. (a) The mean noise input $\sigma_{1} \xi_{1}$ (solid line) to the membrane potential equation (3.1) averaged over $1 \mathrm{~ms}$ bins before the spike. The dot-dash lines indicate the standard uncertainty in the mean. (b) The mean noise input $\sigma_{2} \xi_{2}$ to the recovery variable equation (3.2) (solid line) and its standard uncertainty (dot-dash lines). (c) The same data as (b), plotted on a logarithmic scale. The growth is well fitted by an exponential (straight line).
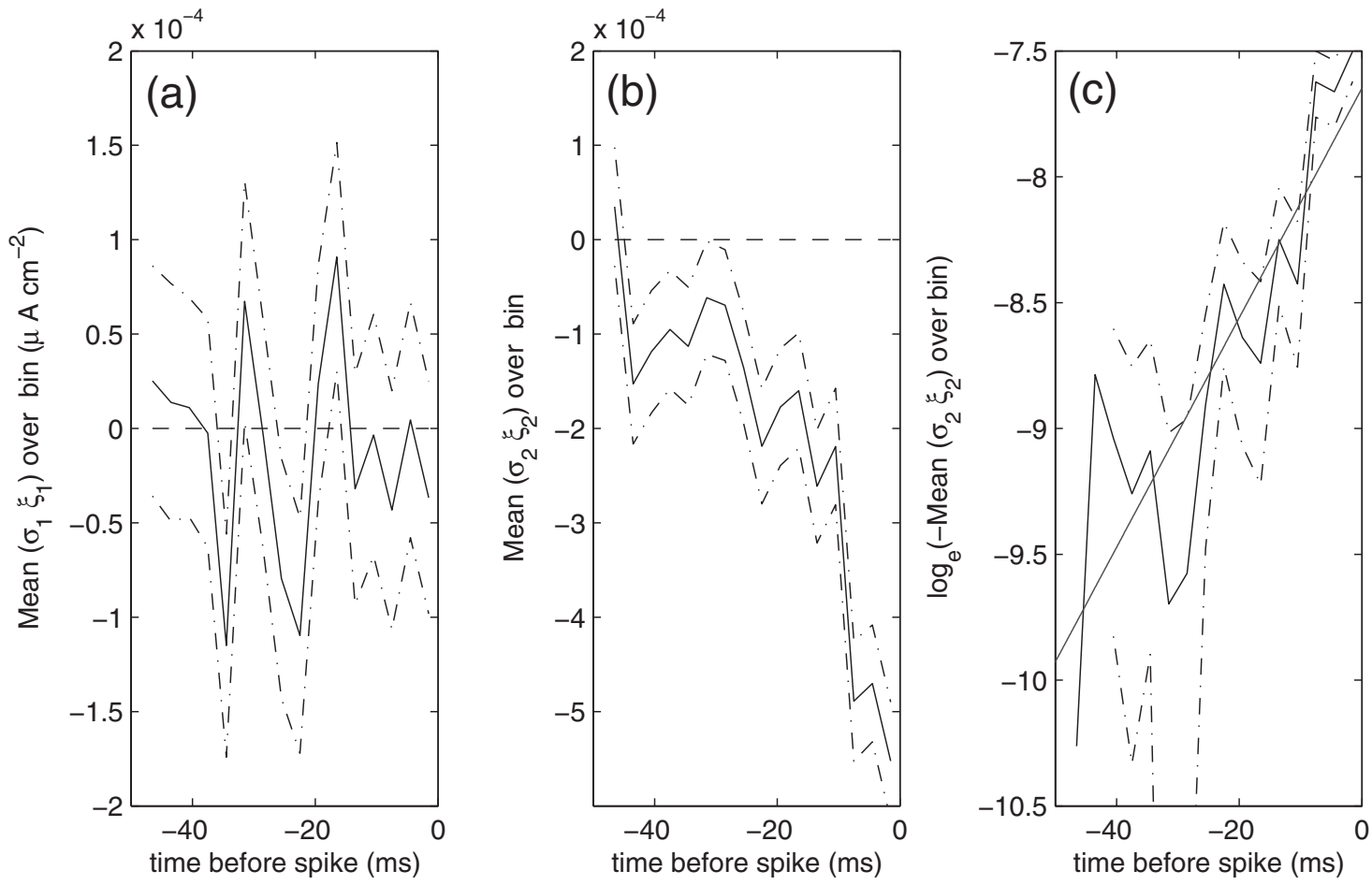

FIG. 8. A plot of the noise input to the Wilson model over the $50 \mathrm{~ms}$ preceding a spike, for the case of $I_{\mathrm{dc}}=21.4 \mu \mathrm{A} \mathrm{cm}{ }^{-2}$ and the lower noise input of $\sigma_{1}=0.015 \mu \mathrm{A} \mathrm{cm}{ }^{-2} \mathrm{~ms}^{1 / 2}$ and $\sigma_{2}=0.015 \mathrm{~ms}^{1 / 2}$. A total of 98 simulations were accepted from 500 attempts. (a) The mean simulated noise input $\sigma_{1} \xi_{1}$ (solid line) to the membrane potential equation (3.1) averaged over $3 \mathrm{~ms}$ bins before the spike. The dot-dash lines indicate the standard uncertainty in the mean. (b) The mean simulated noise input $\sigma_{2} \xi_{2}$ to the recovery variable equation (3.2) (solid line) and its standard uncertainty (dot-dash lines). Note how the reduction in noise input to the recovery variable occurs over a long time-frame before the spike. (c) The same data as (b), plotted on a logarithmic scale. An exponential fit (straight line) has been applied. 
time intervals (1 ms). Parts (a) and (b) show the results for the random inputs to $V$ and $R$, respectively. Part (c) is a plot of the negative of the input to the $R$ equation on a logarithmic scale, to show the exponential nature of the growth.

The graphs show that, in this case, there is a clear reduction in mean noise input to the recovery variable in Eq. (3.2) on the approach to the spike; in other words, we can say that it is the reduction in the mean of these random numbers (that is, the fact that they are on average slightly negative) that has caused $R$ to drop sufficiently for the spike to occur. Note that close to the firing event, the random numbers need to get more negative - this is because with a reduced $R$ there is a stronger drive towards equilibrium that must be overcome until the point where the nonlinear dynamics takes over. There is no obvious trend in the input to $V$, suggesting that the increase in membrane potential on the approach to a spike is best attributed to a reduction in recovery variable (i.e., reduction in inhibition). Once the neuron has fired, the random input returns to its long term average (zero, not shown on the graph). It is important to realize that the mean random input over any time frame will be zero, so long as the time-frame has not been selected in a biased manner. The fact that it is not zero in the case shown here is because the sequences have been time aligned so that the action potentials are generated at the same point in time-i.e., the selection of the time windows is biased.

With reduced noise intensity, we would expect that the required reduction in $R$ in order to produce an action potential will be harder to achieve. Figure 8 shows a similar plot for half the noise level of Fig. 7, indicating that the required increase in negative bias to $R$ occurs over a longer time (in this case the full $48 \mathrm{~ms}$ of the simulation). Conversely, in the limit of large noise, we would expect that a spike could be generated as a result of just a few random numbers being significantly lower than their long term average-i.e., its onset could be extremely rapid. Equations (3.6) and (3.7) effectively demonstrate this by their scaling of the $p_{1}$ and $p_{2}$ terms, respectively. The equation for $\dot{q}_{2}$ contains a term $\left(\sigma_{2} / \tau_{R}\right)^{2} p_{2}$, from which we conclude that a high value of $\sigma_{2}$ will lead to a high sensitivity of $q_{2}=R$ to any changes in $p_{2}$.

\section{E. Shape of action potential}

Finally, we mention the average shape of the action potential as a function of the time taken for the action potential to occur (i.e., inverse spike rate). For the case of $I_{\mathrm{dc}}=21.475 \mu \mathrm{A} \mathrm{cm}^{-2}, \sigma_{1}=0.02 \mu \mathrm{A} \mathrm{cm}^{-2} \mathrm{~ms}^{1 / 2}$, and $\sigma_{2}$ $=0.02 \mathrm{~ms}^{1 / 2}$, we have carried out 40000 simulations over $40 \mathrm{~ms}$. Each simulation is started at the equilibrium value of $V$ and $R$. We then group the trajectories in terms of the time taken for them to reach a threshold of $-55 \mathrm{mV}$, and average over each group. We do the same with the random input to the recovery variable.

In Fig. 9 we show these averaged trajectories and noise inputs. The mean trajectories form an envelope corresponding to a slow time scale. For simulations that reach action potential quickly, the mean membrane potentials rapidly rise from their equilibrium (starting) values until they join this envelope; the mean recovery variables rapidly fall until their
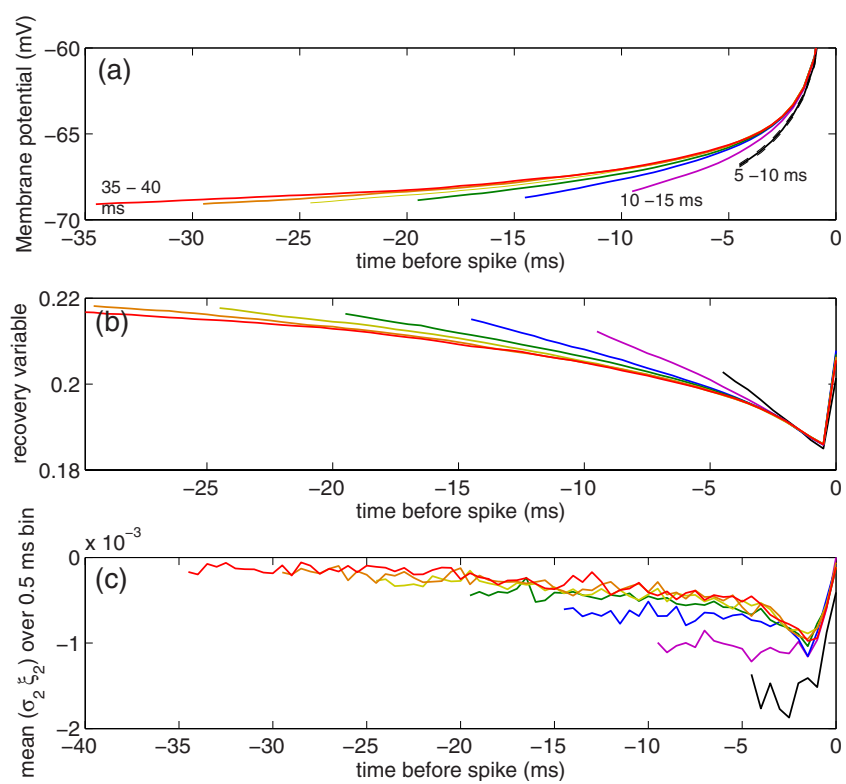

FIG. 9. (Color online) A plot of the mean trajectories for the approach to a firing event, for the case of $I_{\mathrm{dc}}=21.475 \mu \mathrm{A} \mathrm{cm}^{-2}$, $\sigma_{1}=0.02 \mu \mathrm{A} \mathrm{cm}^{-2} \mathrm{~ms}^{1 / 2}$, and $\sigma_{2}=0.02 \mathrm{~ms}^{1 / 2}$. A total of 7759 out of 40000 simulations were accepted. (a) The mean membrane potential, grouped by time taken to fire. The dashed line indicate the standard uncertainties in the mean for one trajectory; the other uncertainties are lower than this. The groups are (in terms of time before firing occurs): $5-10 \mathrm{~ms}$ (black), $10-15 \mathrm{~ms}$ (violet), 15-20 ms (blue), 20-25 ms (green), 25-30 ms (yellow), 30-35 ms (orange), and 35-40 ms (red). Each sequence has been time aligned so they reach the same value of $V$ simultaneously. For the 5-10 ms sequence, only the last $5 \mathrm{~ms}$ have been plotted so that all sequences in the group contribute at every point in the graph; similarly for the other sequences. (b) The mean recovery variable, grouped in a similar way. (c) The mean random input to the recovery variable equation (3.2), over periods of $0.5 \mathrm{~ms}$, with simulations grouped in the same way as part (a).

envelope is reached. This gives rise to an "average" action potential that is sharper in time for an action potential that occurs soon after simulation starts. In principle, this is a prediction that can be tested experimentally, though the analysis only applies to subthreshold neurons (i.e., neurons not in a limit-cycle behavior, but ones whose firing is triggered by noise input). Experimentally, the shape of the action potential as a function of time since the last action potential can be examined. For example, we would expect that action potentials which occur soon after their predecessor will have risen very quickly; moreover, we could expect an exponential shift in potential for spikes that have occurred a long time after the previous one.

The mean noise input is also shown in the figure, demonstrating that, for the case of neurons that fire an action potential quickly, the average random input to the recovery variable has been considerably lower than its long term average over this short period of time. Conversely, neurons that have taken longer to fire, have "accumulated" this reduction in inhibitory input gradually over a much longer period of time. However, in all cases, there must be a similar drop in the random input in the $2 \mathrm{~ms}$ preceding the spike. In terms of 

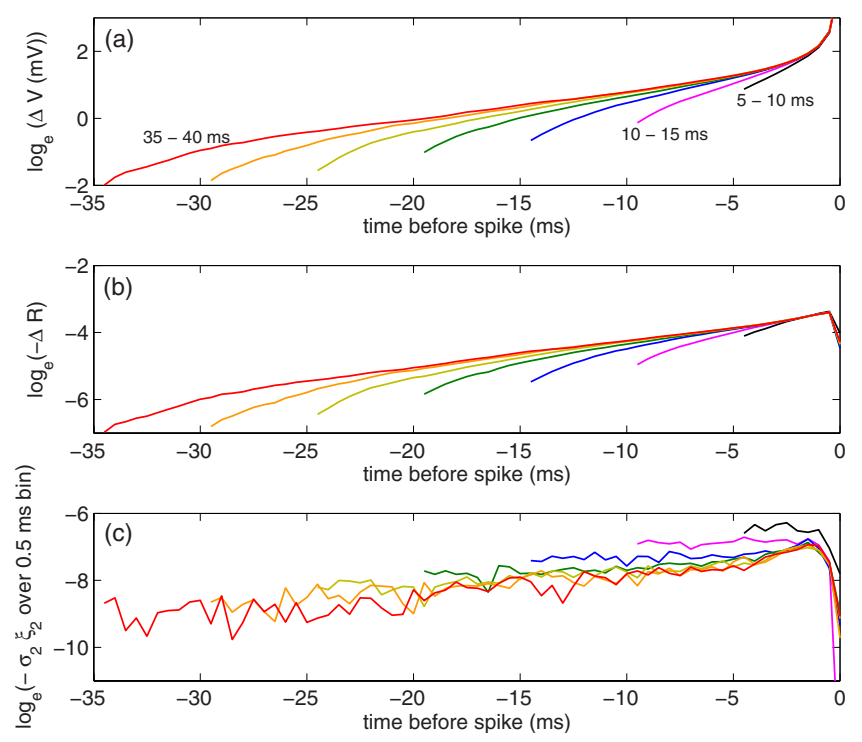

FIG. 10. (Color online) A plot of the mean trajectories for the approach to a firing event, in terms of the deviation from equilibrium values, for the same case as Fig. 9. (a) The natural logarithm of the mean deviation of the membrane potential from its equilibrium value, grouped by time taken to fire. The groups are (in terms of time before firing occurs): 5-10 ms (black), 10-15 ms (violet), 15-20 ms (blue), 20-25 ms (green), 25-30 ms (yellow), 30-35 ms (orange), and 35-40 ms (red). Each sequence has been time aligned so they reach the same value of $V$ simultaneously. For the 5-10 ms sequence, only the last $5 \mathrm{~ms}$ have been plotted so that all sequences in the group contribute at every point in the graph; similarly for the other sequences. (b) The natural logarithm of the (negative) of the deviation of the mean recovery variable from its equilibrium value, grouped in a similar way. (c) The natural logarithm of the (negative) mean random input to the recovery variable equation (3.2), over periods of $0.5 \mathrm{~ms}$, with simulations grouped in the same way as part (a).

$\vec{p}$, this means that a small initial value of $\vec{p}$ in the Hamiltonian approach will represent a trajectory that takes a long time to fire (i.e., the negative bias in $p_{2}$ accumulates more slowly), whereas a high initial value of $\vec{p}$ gives rise to a rapidly firing trajectory.

Figure 10 shows the same information in a logarithmic form. From this graph we see that the limiting envelope for the change in the membrane potential and recovery variables from their equilibrium values $\Delta V$ and $\Delta R$, respectively, are approximately exponentials. Also, we see a similar behavior in the noise input to the recovery variable-in the limit of large times before the spike, the noise term changes in an exponential manner. The growth rates for the potential and recovery processes are similar, namely, around $0.08 \mathrm{~ms}^{-1}$. These are higher than the slow eigenvalue of the matrix $M$ calculated numerically from Eqs. (3.8) and (3.9) or, equivalently, the Jacobian matrix of the linearized equations (3.1) and (3.2), for a driving current of $21.475 \mu \mathrm{A} \mathrm{cm}^{-2}$, which is $0.020 \mathrm{~ms}^{-1}$.

In order to reproduce the slow timescale of the matrix $M$ we have had to reduce the noise input to $0.005 \mu \mathrm{A} \mathrm{cm}^{-2} \mathrm{~ms}^{1 / 2}$ and $0.005 \mathrm{~ms}^{1 / 2}$ for $\sigma_{1}$ and $\sigma_{2}$, respectively, and increase the time of simulation. Lower noise en-
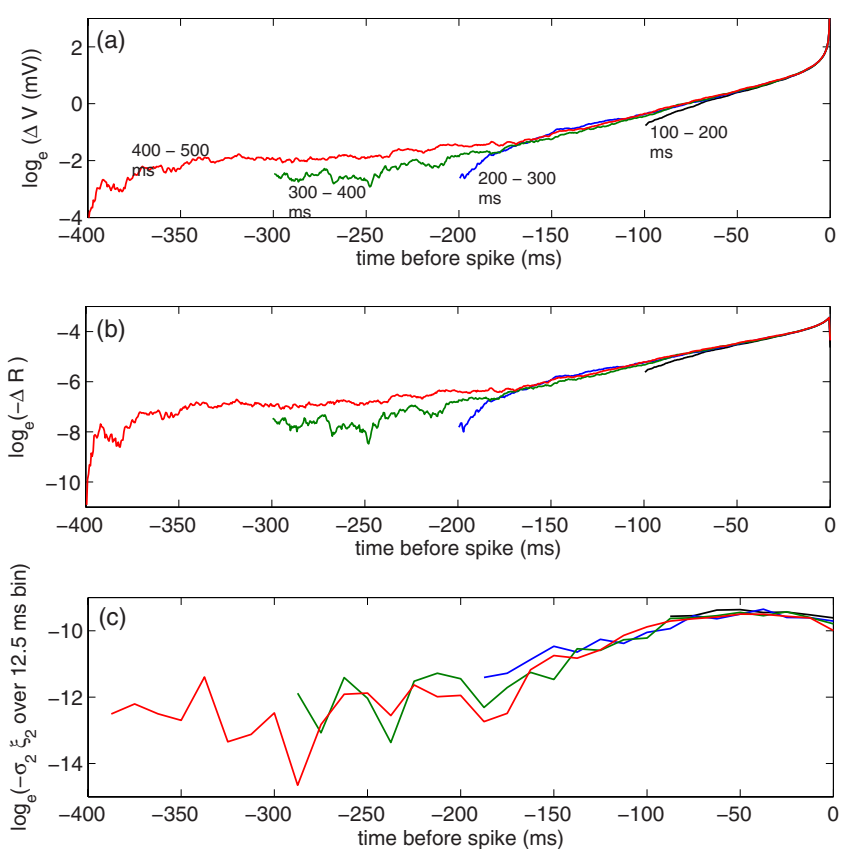

FIG. 11. (Color online) A plot of the mean trajectories for the approach to a firing event, in terms of the deviation from equilibrium values, for the case of $I_{\mathrm{dc}}=21.475 \mu \mathrm{A} \mathrm{cm}^{-2} \mathrm{~ms}^{1 / 2}, \sigma_{1}$ $=0.005 \mu \mathrm{A} \mathrm{cm}^{-2} \mathrm{~ms}^{1 / 2}$, and $\sigma_{2}=0.005 \mathrm{~ms}^{1 / 2}$. A total of 1509 out of 10000 simulations were accepted. (a) The natural logarithm of the mean deviation of the membrane potential from its equilibrium value, grouped by time taken to fire. The groups are (in terms of time before firing occurs): $100-200 \mathrm{~ms}$ (black), 200-300 ms (blue), 300-400 ms (green), and 400-500 ms (red). Each sequence has been time-aligned so they reach the same value of $V$ simultaneously. (b) The natural logarithm of the (negative) of the deviation of the mean recovery variable from its equilibrium value, grouped in a similar way. (c) The natural logarithm of the (negative) mean random input to the recovery variable equation (3.2), with simulations grouped in the same way as part (a). Note that this plot exhibits a region of exponential growth with rate constant $0.020 \mathrm{~ms}^{-1}$, between 200 and $100 \mathrm{~ms}$ before the spike, in agreement with the slow eigenvalue of matrix $M$.

sures that the subthreshold dynamics are more linear in nature, so that Eq. (2.35) is more applicable. The same driving current is used. In this situation, there is a very low probability of the neuron firing, and in order to sample enough cases for good statistics, we have required a run time of about 60 hours on an Intel Xeon processor (with clock speed of $2.7 \mathrm{GHz}$ ). In this case just 1509 out of a total of 10000 simulations resulted in a firing within $500 \mathrm{~ms}$. This time frame is arguably at the limit of what might be considered plausible for a gap between neuron firings during normal cortical behavior. Figure 11 shows the manner in which $\Delta V$, $\Delta R$ and the noise input to the recovery process vary with time as the spike is approached. In this case we observe that the noise term also shows a region of exponential growth with a similar gradient of $0.020 \mathrm{~ms}^{-1}$, in agreement with the prediction of Eq. (2.35). However, the gradients of the $V$ and $R$ processes are not quite what we would expect, being about $25 \%$ too low. This may indicate that the small fluctuations approximation is still not valid. 


\section{CONCLUSIONS}

We have developed Lagrangian and Hamiltonian descriptions of a stochastic process in $N$ dimensions, and applied the Hamiltonian form to the stochastic neuron model of Wilson. While a Lagrangian analysis in the manner of Paninski [1] is likely to be a better approach for analyzing membrane potential fluctuations, the Hamiltonian form has allowed us to give a physical interpretation to the neuron's behavior on the approach to an action potential. The conjugate momenta variables are linear combinations of the noise inputs to the neuron; the theory predicts that the magnitudes of these increase exponentially on the approach to a neural firing event, which we have confirmed by simulation. Particularly, we find that an exponentially increasing negative input to the inhibitory recovery process is responsible for the firing event, in agreement to the experimental results of Rudolph et al. [6]. In terms of the Hamiltonian description, we can therefore consider an action potential as being the result of an exponentially growing conjugate momentum pulling the system away from a stable equilibrium into a nonlinear regime.
[1] L. Paninski, J. Comput. Neurosci. 21, 71 (2006).

[2] L. Badel, W. Gerstner, and M. J. E. Richardson, Neurocomputing 69, 1062 (2006).

[3] L. Badel, W. Gerstner, and M. J. E. Richardson, Phys. Rev. E 78, 011914 (2008).

[4] L. Ingber, Phys. Rev. E 49, 4652 (1994).

[5] M. Pospischil, Z. Piwkowska, M. Rudolph, T. Bal, and A. Destexhe, J. Neurophysiol. 97, 2544 (2007).

[6] M. Rudolph, M. Pospischil, I. Timofeev, and A. Destexhe, J. Neurosci. 27, 5280 (2007).

[7] D. A. Steyn-Ross, M. L. Steyn-Ross, M. T. Wilson, and J. W. Sleigh, Phys. Rev. E 74, 051920 (2006).

[8] H. R. Wilson, Spikes, Decisions, and Actions: The Dynamical Foundations of Neuroscience (Oxford University Press, Oxford, 1999).

[9] H. Goldstein, Classical Mechanics, 2nd ed. (Addison-Wesley, Reading, MA, 1980).
[10] L. Ingber, Phys. Rev. E 55, 4578 (1997)

[11] M. T. Wilson, M. L. Steyn-Ross, D. A. Steyn-Ross, and J. W. Sleigh, Phys. Rev. E 72, 051910 (2005).

[12] S. Chaturvedi, C. W. Gardiner, I. S. Matheson, and D. F. Walls, J. Stat. Phys. 17, 469 (1977).

[13] A. L. Hodgkin and A. F. Huxley, J. Physiol. (London) 117, 500 (1952).

[14] J. Rinzel, Fed. Proc. 37, 2793 (1985).

[15] R. Fitzhugh, Biophys. J. 1, 445 (1961).

[16] J. S. Nagumo, S. Arimoto, and S. Yoshizawa, Proc. IRE 50, 2061 (1962).

[17] P. E. Kloeden and E. Platen, Numerical Solution of Stochastic Differential Equations (Springer, Berlin, 1992).

[18] A. Destexhe, M. Rudolph, J.-M. Fellous, and T. J. Sejnowski, Neuroscience 107, 13 (2001).

[19] http://cvr.yorku.ca/webposes/book.html 